\title{
Experimental approximation of breast tissue permittivity and conductivity using NN- based UWB imaging
}

\begin{abstract}
This paper presents experimental study to distinguish between malignant and benign tumors in early breast cancer detection using Ultra Wide Band (UWB) imaging. The contrast between dielectric properties of these two tumor types is the main key. Mainly water contents control the dielectric properties. Breast phantom and tumor are fabricated using pure petroleum jelly and a mixture of wheat flour and water respectively. A complete system including Neural Network (NN) model is developed for experimental investigation. Received UWB signals through the tumor embedded breast phantom are fed into the NN model to train, test and determine the tumor type. The accuracy of the experimental data is about 98.6\% and $99.5 \%$ for permittivity and conductivity respectively. This leads to determine tumor dielectric properties accurately followed by distinguish between malignant and benign tumors. As malignant tumors need immediate further medical action and removal, this findings could contribute to save precious file in near future.
\end{abstract}

Keyword: Breast cancer; Neural network; Breast tissues dielectric properties 